\title{
A Biomechanical Model of Muscle Contraction
}

\author{
J. Bestel, F. Clément, and M. Sorine
}

INRIA Rocquencourt, 78153 Le Chesnay Cedex, France

\begin{abstract}
Models of the electro-mechanical activity of the cardiac muscle can be very useful in computing stress, strain and action potential fields from three-dimensional image processing. We designed a chemically-controlled constitutive law of cardiac myofibre mechanics, acting on the mesoscopic scale and devoted to be embedded into a macroscopic model. This law ensues from the modelling of the collective behaviour of actin-myosin molecular motors, acting on the nanoscopic scale to convert chemical into mechanical energy. The resulting dynamics of sarcomeres, acting on the microscopic scale, is shown to be consistent with the "sliding filament hypothesis", which was first introduced by A. F. Huxley [1].
\end{abstract}

\section{Excitation-Contraction Model on the Myofibre Scale}

The contractile elements (CE) of myofibres are able to produce stress while shortening in response to a chemical input $u(t)$, mainly depending on the calcium concentration. We propose, as a constitutive relation in the myofibre direction between stress $\sigma$ and strain $\varepsilon$, the following set of controlled differential equations (modified from [2], 3]) of visco-elasto-plastic type:

$$
\left\{\begin{array}{llrl}
\dot{k}_{c}=-(|u|+|\dot{\varepsilon}|) & k_{c}+k_{0}|u|_{+} & k_{c}(0) & =\sigma_{c}(0)=0 \\
\dot{\sigma}_{c}=-(|u|+|\dot{\varepsilon}|) & \sigma_{c}+k_{c} \dot{\varepsilon}+\sigma_{0}|u|_{+} & \sigma & =k_{c} \xi_{0}+\sigma_{c}+\eta \dot{\varepsilon}
\end{array}\right.
$$

The system is able to account for : shortening from resting conditions $(\dot{\varepsilon}=0)$ in response to $u(t)$, passive behaviour of CE $(u=0)$ as described in [4, static relation between $\varepsilon$ and $\sigma$, and isotonic contraction $\left(\dot{\sigma}_{c}=0\right.$ and $\left.\dot{\varepsilon}<0\right)$ as in Hill's experimental model. For the purpose of ultrasonographic-image processing, the system is further being embedded into a partial differential equation model including reaction-diffusion equations to rule the propagation of action potential.

\section{Excitation-Contraction Model on the Sarcomere Scale}

On a microscopic scale, the sarcomere is made up of thin and thick parallel filaments. When ATP is available and the intracellular level of calcium reaches a threshold, myosin heads of the thick filament become likely to bind actin sites on the thin filament. The elastic free energy $W$ of the actin-myosin interaction is responsible for muscle contraction: stress response to strain causes relative sliding of the actin over the myosin filament. Let $n(\xi, t)$ be the density of crossbridges with strain $\xi$ at time $t$ and $\varepsilon$ the strain (normalized by the ratio of rest to maximal length of cross-bridges). Then, Huxley's model is:

$$
\partial_{t} n+\dot{\varepsilon} \partial_{\xi} n=f(1-n)-g n, \quad \sigma(t)=-\int_{-\infty}^{+\infty} \partial_{\xi} W(\xi) n(\xi, t) d \xi+\eta \dot{\varepsilon}
$$


The following choice of $f(\xi, t)$ and $g(\xi, t)$, the positive rates at which crossbridges respectively fasten and unfasten, is consistent with myofibre behaviour:

$f(\xi, t)=|u(t)|_{+}$for $\xi \in[0,1](=0$ elsewhere $), g(\xi, t)=|u(t)|+|\dot{\varepsilon}(t)|-f(\xi, t)$

In the particular case $-\partial_{\xi} W(\xi)=k_{0} \xi_{0}+\sigma_{0} \xi$, we notice, as in [5], that the total stiffness $k_{c}$ and total elastic stress $\sigma_{c}$ are respectively proportional to the zero and first-order moment of $n$ :

$$
k_{c}(t)=k_{0} \int_{-\infty}^{+\infty} n(\xi, t) d \xi \quad \text { and } \quad \sigma_{c}(t)=\sigma_{0} \int_{-\infty}^{+\infty} \xi n(\xi, t) d \xi
$$

where $k_{0}$ and $\sigma_{0}$ are constants related to physical parameters of crossbridges. The system (1) is a set of equations of moments derived from (2), (3) and (4).

\section{Excitation-Contraction on the Molecular-Motor Scale}

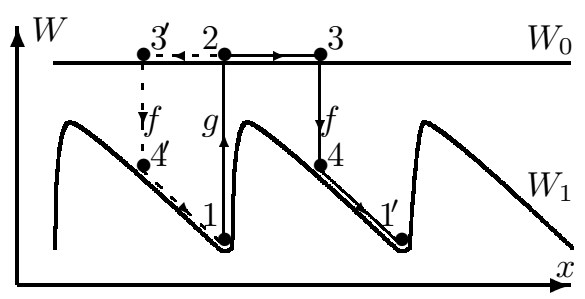

$1\left(1^{\prime}\right) . \mathrm{AM}+\mathrm{ATP} \rightarrow \mathrm{M} \cdot \mathrm{ATP}+\mathrm{A}$

g. Myosin unbinding

2. M.ATP $\rightarrow$ M.ADP.P

Myosin free motion

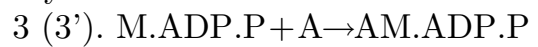

$f$. Myosin binding controlled by $\mathrm{Ca}^{2+}$ $4\left(4^{\prime}\right)$. AM.ADP.P $\rightarrow \mathrm{AM}+\mathrm{ADP}+\mathrm{P}$

Myosin sliding on actin

Two-state model for a single motor [6] and Four-phase ATP-cycle

The motion of a myosin head is described by a Langevin equation with $\eta$, friction coefficient, $b$, thermal normalized gaussian white noise, and $F_{\text {ext }}$, external force:

$$
\eta \dot{x}=-\partial_{x} W_{i}(x)+\sqrt{2 \eta k_{B} T} b(t)+F_{\text {ext }}, \quad i=0,1
$$

The myosin is likely to move one way, as the thermal fluctuations are rectified by the periodic "sawtooth" potential $W_{1}$ (probable moves are $1 \rightarrow 1$ and $1 \rightarrow 1^{\prime}$ ). Collective behaviour of motors [6]. Applying the Fokker-Planck formalism to (5), $n(t, \xi)$ appears as the average density of myosin heads bound a distance $\xi$ away from the nearest local minimum of $W_{1}$.

\section{Conclusion and Perspectives}

We have proposed a controlled contraction model on the myofibre scale consistent with models designed on the sarcomere and molecular scales. A more precise model accounting for detailed $\mathrm{Ca}^{2+}$ action is under development. 


\section{References}

[1] A. F. Huxley. Muscle structure and theories of contraction. In Progress in biophysics and biological chemistry, volume 7. Pergamon Press, 1957.

[2] J. Bestel. PhD thesis, Université Paris 9, 2000.

[3] J. Bestel and M. Sorine. A differential model of muscle contraction and applications. In Schloessmann Seminar on Mathematical Models in Biology, Chemistry and Physics, Max Plank Society, Bad Lausick, Germany, May 19-23 2000.

[4] I. Mirsky and W. W. Parmley. Cardiac Mechanics: Physiological, Clinical, and Mathematical Considerations, chapter 4. J. Wiley, 1974.

[5] G. I. Zahalak. A distribution-moment approximation for kinetic theories of muscular contraction. Mathematical Biosciences, (114):55:89, 1981.

[6] F. Jülicher, A. Ajdari, and J. Prost. Modeling molecular motors. Reviews of Modern Physics, 69(4), October 1997. 